\title{
Non-alcoholic fatty liver disease fibrosis score predicts hematological toxicity of chemotherapy including irinotecan for colorectal cancer
}

\author{
MASASHI YAHAGI, MASASHI TSURUTA, HIROTOSHI HASEGAWA, KOJI OKABAYASHI and YUKO KITAGAWA \\ Department of Surgery, Keio University School of Medicine, Tokyo 160-8582, Japan
}

Received November 17, 2016; Accepted January 13, 2017

DOI: $10.3892 / \operatorname{mco} .2017 .1177$

\begin{abstract}
Liver dysfunction that may affect drug metabolism is a major concern in patients treated with chemotherapy. Thus, assessment of the degree of liver dysfunction is crucial for predicting the adverse events of chemotherapy. The non-alcoholic fatty liver disease fibrosis score (NFS) is a non-invasive clinical scoring system constructed from routine clinical and laboratory variables. The aim of this study was to evaluate whether NFS was useful for predicting the adverse events of chemotherapy including irinotecan (CPT-11) for colorectal cancer. Between January, 2007 and May, 2013, a total of 87 patients with unresectable/recurrent colorectal cancer who received first-line chemotherapy including CPT-11 were reviewed. Demographic variables, including pretreatment NFS, were retrospectively collected from medical records. The primary outcome was the association between pretreatment NFS and adverse events, such as hematological and non-hematological toxicity, of chemotherapy including CPT-11. The median pretreatment NFS was 1.302 (range, 5.158-2.620). Pretreatment NFS was an independent risk factor for hematological toxicity in a multivariate analysis (coefficient $=0.932$, 95\% CI: 0.083-1.781; $\mathrm{P}=0.031$ ). Receiver operating characteristic curve analysis identified 0.347 as the optimal cut-off value associated with hematological toxicity. Using this cut-off, high NFS was found to be a significant risk factor for hematological toxicity (coefficient $=2.019,95 \% \mathrm{CI}$ : 0.239-3.798, $\mathrm{P}=0.026$ ), but not for non-hematological toxicity $(\mathrm{P}=0.546)$. Therefore, based on these results, NFS appears to be a significant predictor of hematological adverse events in chemotherapy including CPT-11 for colorectal cancer and it is a non-invasive, useful tool that may be used for determining regimens or doses of chemotherapy including CPT-11.
\end{abstract}

Correspondence to: Dr Masashi Tsuruta, Department of Surgery, Keio University School of Medicine, 35 Shinanomachi, Shinjyuku-ku, Tokyo 160-8582, Japan

E-mail: championtsuru@yahoo.co.jp

Key words: non-alcoholic fatty liver disease fibrosis score, colorectal cancer, hematological toxicity, non-hematological toxicity, irinotecan

\section{Introduction}

Liver dysfunction, which may affect drug metabolism, is a major concern in patients receiving chemotherapy for colorectal cancer. Thus, the assessment of the degree of any liver dysfunction, such as hepatic fibrosis and inflammation, is crucial for predicting the effects or adverse events of chemotherapy. Currently, the gold standard method for the assessment of liver dysfunction is liver biopsy; however, this is a costly and invasive method, with a high risk of complications. In addition, the typical sample is small and may result in sampling error $(1,2)$. Certain benefits of non-invasive clinical scoring systems, constructed from routine clinical and laboratory variables, have been reported for several liver conditions, including non-alcoholic fatty liver disease (NAFLD) (3). Among those, the NAFLD fibrosis score (NFS) has been recommended even for Japanese populations, in which the incidence of obesity is relatively low $(4,5)$.

Irinotecan (CPT-11) is a key drug used to treat unresectable/recurrent colorectal cancer. Polymorphisms in the uridine diphosphate-glucuronosyltransferase (UGT)1A1 gene, which is involved in the metabolism of CPT-11 in the liver, may cause severe adverse events, such as neutropenia (6). However, testing for such polymorphisms is time-consuming and costly. In addition, the number of UGT1A1*28 homozygous patients who experience severe adverse events due to CPT-11 is quite small in the Japanese population. Thus, it is important to identify a more appropriate surrogate marker reflecting liver dysfunction in chemotherapy with CPT-11. The aim of this study was to evaluate whether NFS is useful for predicting the effects and adverse events of chemotherapy including CPT-11 for colorectal cancer.

\section{Materials and methods}

Patients and clinicopathological characteristics. Between January, 2007 and May, 2013, 118 patients were diagnosed with unresectable/recurrent colorectal cancer at Keio University Hospital (Tokyo, Japan), of whom 87 patients who underwent first-line chemotherapy including CPT-11 were enrolled in this study.

The tumorlocation, from the vermiform appendix (VA) to the rectum, was classified according to the 7th edition of the TNM Classification of Malignant Tumours (http://www.uicc.org/ 
sites/main/files/private/TNM_Classification_of_Malignant_ Tumours_Website_15\%20MAy2011.pdf). The colon was defined as spanning from the cecum to the sigmoid colon, while 'other' was defined as the rectum; thus, tumors of the VA were excluded. In terms of histological types, well- and moderately differentiated tubular adenocarcinomas were defined as highly differentiated carcinomas, whereas poorly differentiated, mucinous adenocarcinomas and signet-ring cell carcinomas were defined as poorly differentiated carcinomas.

Demographic variables were retrospectively collected from medical records and included age, gender, tumor location, histological type, number of metastatic sites (single or multiple), temporal association of metastasis (synchronous or metachronous), cycles of chemotherapy, use of bevacizumab, pretreatment total bilirubin serum levels and pretreatment NFS. NFS was calculated as follows: NFS $=1.675+0.037 \mathrm{x}$ age (years) $+0.094 \mathrm{x}$ BMI $\left(\mathrm{kg} / \mathrm{m}^{2}\right)+1.13 \mathrm{x}$ impaired fasting glucose/diabetes (yes $=1$, no $=0)+0.99 x$ AST/ALT ratio- $0.013 x$ platelet $\left(x 10^{9} / 1\right)-0.66 x$ albumin $(\mathrm{g} / \mathrm{dl})$, where BMI, body mass index; AST, aspartate aminotransferase; and ALT, alanine aminotransferase.

Treatment and evaluation. Our regimen including CPT-11 was FOLFIRI or uracil/ftorafur/leucovorin combined with CPT-11 (TEGAFIRI); thus, the participants received FOLFIRI(CPT-11 $150 \mathrm{mg} / \mathrm{m}^{2}$ on day 1 , leucovorin $200 \mathrm{mg} / \mathrm{m}^{2}$ on day 1,5 -fluorouracil $400 \mathrm{mg} / \mathrm{m}^{2}$ on day 1,5 -fluorouracil $2,400 \mathrm{mg} / \mathrm{m}^{2}$ as a 46-h infusion, every 2 weeks) or TEGAFIRI (uracil/ftorafur $300 \mathrm{mg} / \mathrm{m}^{2} /$ day on days $1-21$, leucovorin $75 \mathrm{mg} /$ day on days $1-21, \mathrm{CPT}-11150 \mathrm{mg} / \mathrm{m}^{2} /$ day on days 1 and 15 , every 4 weeks). The National Cancer Institute Common Terminology Criteria for Adverse Events (CTCAE), version 4.0, were used to assess toxicity (7).

Treatment was administered until disease progression, development of unacceptable toxicity, or the patient's wish to withdraw. The doses of CPT-11 were lowered by $20 \%$ if severe toxic effects (CTCAE grade $\geq 3$ ) occurred. Patients were evaluated for progression every four cycles by computed tomography. During the follow-up period, any continuing adverse effects were closely monitored.

The primary outcome was the association between pretreatment NFS and adverse events, such as hematological and non-hematological toxicity, of chemotherapy including CPT-11.

This study was approved by the Ethics Review Board of our institute (no. 20140459).

Statistical methods. Logistic regression analysis was used to identify univariate and multivariate risk factors for hematological and non-hematological toxicity (CTCAE grade $\geq 3$ ). The coefficient (coef) was used to estimate the impact on hematological and non-hematological toxicity. All predictors with $\mathrm{P}<0.05$ in univariate analysis and clinically important factors were retained in the multivariate models.

A receiver operating characteristic (ROC) curve analysis was performed to determine a cut-off point for NFS as a continuous variable. Youden's index was calculated and the maximum value was used as the optimum cut-off point. Using this cut-off value, the impact on adverse events was also assessed. Each risk factor was assessed using the $\chi^{2}$ test, Mann-Whitney U test, Fisher's exact test and logistic regression analysis. All statistical analyses were two-sided and
Table I. Patient characteristics.

\begin{tabular}{lc}
\hline Variables & All (n=87) \\
\hline Gender (male/female) & $59 / 28$ \\
Age, years & $67(36-82)^{\mathrm{a}}$ \\
Tumor location (C/A/T/D/S/R) & $5 / 15 / 9 / 6 / 24 / 28$ \\
Histological type (well/poorly & $77 / 10$ \\
differentiated) & \\
Single/multiple metastasis & $49 / 38$ \\
Synchronous/metachronous & $37 / 50$ \\
metastasis & $13(1-126)^{\mathrm{a}}$ \\
Number of cycles & $45 / 42$ \\
Use of bevacizumab (yes/no) & $0.6(0.3-1.5)^{\mathrm{a}}$ \\
Total serum bilirubin ${ }^{\mathrm{b}}, \mathrm{mg} / \mathrm{dl}$ & 6 \\
NFS & 1.5 .158 to 2.62$)^{\mathrm{a}}$ \\
Hematological toxicity & 6 \\
Decreased platelet count & 1 \\
Decreased neutrophil count & 5 \\
Non-hematological toxicity & 13 \\
Vomiting & 5 \\
Diarrhea & 4 \\
Nervous system disorders & 1 \\
Increased serum bilirubin & 1 \\
Cough & 2 \\
\hline
\end{tabular}

${ }^{a}$ Median (range). ${ }^{b}$ Pretreatment. ${ }^{\mathrm{c} C o m m o n}$ Terminology Criteria for Adverse Effects grade $\geq 3$. C, cecum; A, ascending colon; T, transverse colon; D, descending colon; S, sigmoid colon; R, rectum; NFS, non-alcoholic fatty liver disease fibrosis score.

statistical significance was set at $\mathrm{P}<0.05$. Statistical analyses were performed using the STATA software, version 11; (StataCorp LP, College Station, TX, USA).

\section{Results}

Patient background. The clinicopathological demographics of the 87 patients are summarized in Table I. The median age was 67 years (range, $36-82$ years) and 59 of the participants (67.8\%) were men. The tumors were located in the colon in 59 patients $(67.8 \%)$. The median pretreatment total bilirubin serum levels were $0.6 \mathrm{mg} / \mathrm{dl}$ (range, $0.3-1.5 \mathrm{mg} / \mathrm{dl}$ ) and the median NFS was 1.302 (range, 5.158-2.620). Grade $\geq 3$ hematological and non-hematological toxicities occurred in $6(6.9 \%)$ and 13 (14.9\%) patients, respectively.

Association between NFS and adverse events. In the univariate analysis, pretreatment NFS was significantly associated with hematological toxicity (Table II). Furthermore, pretreatment NFS was an independent risk factor for hematological toxicity in a multivariate analysis (coef $=0.932,95 \%$ CI: 0.083-1.781; $\mathrm{P}=0.031$ ), adjusted for gender, age, temporal association of metastasis, cycle number and pretreatment total bilirubin serum levels. ROC analysis, including Youden's index of NFS, identified 0.347 as the optimal cut-off value associated with hematological toxicity (Fig. 1). Using this cut-off, 21 (24.1\%) 
Table II. Uni- and multivariate analysis for hematological toxicity.

\begin{tabular}{lccc}
\hline Variables & Unadjusted coef $(95 \%$ CI $)$ & P-value & Adjusted coef (95\% CI) \\
\hline Male gender & $-1.558(-3.321$ to 0.205$)$ & 0.083 & $-1.247(-3.480$ to 0.985$)$ \\
Age & $0.173(-0.068$ to 0.103$)$ & 0.691 & $0.008(-0.131$ to 0.147$)$ \\
Rectum & $0.056(-1.704$ to 1.816$)$ & 0.950 & 0.274 \\
Multiple metastasis & $-0.470(-2.223$ to 1.283$)$ & 0.599 & \\
Synchronous metastasis & $-1.386(-3.578$ to 0.805$)$ & 0.215 & $-0.664(-3.504$ to 2.175$)$ \\
Number of cycles & $0.016(-0.016$ to 0.049$)$ & 0.320 & $0.022(-0.015$ to 0.058$)$ \\
Use of bevacizumab & $-0.074(-1.733$ to 1.584$)$ & 0.930 & 0.647 \\
Total serum bilirubin ${ }^{\text {a }}$ & $1.248(-1.619$ to 4.116$)$ & 0.394 & $-0.434(-4.516$ to 3.648$)$ \\
NFS $^{\text {a }}$ & $0.957(0.257$ to 1.658$)$ & 0.007 & $0.932(0.083$ to 1.781$)$
\end{tabular}

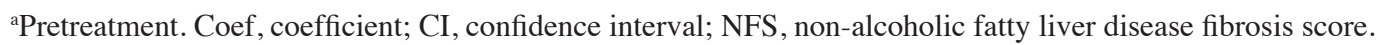

Table III. Patient characteristics by NFS.

\begin{tabular}{|c|c|c|c|}
\hline Variables & High NFS $(n=21)$ & Low NFS $(n=66)$ & P-value \\
\hline Gender (male/female) & $13 / 8$ & $46 / 20$ & $0.506^{\mathrm{b}}$ \\
\hline Age, years & $73(47-82)^{\mathrm{a}}$ & $65(36-80)^{a}$ & $0.007^{\mathrm{c}}$ \\
\hline Tumor location (colon/rectum) & $16 / 5$ & $43 / 23$ & $0.346^{\mathrm{b}}$ \\
\hline \multicolumn{4}{|l|}{ Histological type } \\
\hline (well/poorly differentiated) & $20 / 1$ & $57 / 9$ & $0.267^{\mathrm{b}}$ \\
\hline Single/multiple metastasis & $14 / 7$ & $35 / 31$ & $0.272^{\mathrm{b}}$ \\
\hline Synchronous/metachronous metastasis & $7 / 14$ & $30 / 36$ & $0.328^{\mathrm{b}}$ \\
\hline Number of cycles & $17(3-87)^{\mathrm{a}}$ & $12(1-126)^{\mathrm{a}}$ & $0.042^{\mathrm{c}}$ \\
\hline Use of bevacizumab (yes/no) & $12 / 9$ & $33 / 33$ & $0.568^{\mathrm{b}}$ \\
\hline Total serum bilirubin ${ }^{\mathrm{e}}, \mathrm{mg} / \mathrm{dl}$ & $0.7(0.5-1.3)^{\mathrm{a}}$ & $0.6(0.3-1.5)^{\mathrm{a}}$ & $0.163^{\mathrm{c}}$ \\
\hline \multirow[t]{2}{*}{$\mathrm{NFS}^{\mathrm{e}}$} & 0.823 & -1.692 & \multirow[t]{2}{*}{$<0.001^{\mathrm{c}}$} \\
\hline & $(-0.268 \text { to } 2.62)^{\mathrm{a}}$ & $(-5.158 \text { to }-0.347)^{\mathrm{a}}$ & \\
\hline Hematological toxicity ${ }^{\mathrm{f}}$ & 4 & 2 & \multirow[t]{3}{*}{$0.028^{\mathrm{d}}$} \\
\hline Decreased platelet count & 1 & 0 & \\
\hline Decreased neutrophil count & 3 & 2 & \\
\hline Non-hematological toxicity ${ }^{\mathrm{f}}$ & 4 & 9 & $0.546^{\mathrm{d}}$ \\
\hline Vomiting & 1 & 4 & \\
\hline Diarrhea & 1 & 3 & \\
\hline Nervous system disorders & 0 & 1 & \\
\hline Increased serum bilirubin & 1 & 0 & \\
\hline Cough & 1 & 1 & \\
\hline
\end{tabular}

${ }^{\mathrm{a}}$ Median (range). ${ }^{\mathrm{b}} \chi^{2}$ test. ${ }^{\mathrm{c}}$ Mann-Whitney U test. ${ }^{\mathrm{d}}$ Logistic regression analysis. ${ }^{\mathrm{e}}$ Pretreatment. ${ }^{\mathrm{f}}$ Common Terminology Criteria for Adverse Effects grade $\geq 3$. NFS, non-alcoholic fatty liver disease fibrosis score.

patients were classified as high NFS, while $66(75.9 \%)$ patients had a low NFS (Table III). High NFS was found to be a significant risk factor for hematological toxicity (coef $=2.019$, 95\% CI: 0.239-3.798; $\mathrm{P}=0.026)$ but not for non-hematological toxicity $(\mathrm{P}=0.546)$. This model exhibited a sensitivity of $83.3 \%$, a specificity of $79.3 \%$, a likelihood ratio for a positive finding of 3.97 , and a likelihood ratio for a negative finding of 0.21. However, pretreatment NFS was not identified as a risk factor for non-hematological toxicity in any univariate or multivariate analysis (Table IV). The results were similar even when the investigation was limited to diarrhea among non-hematological toxicities (coef $=0.214,95 \% \mathrm{CI}$ : -0.514 to 0.942; $\mathrm{P}=0.564)$.

\section{Discussion}

Our findings indicate that NFS may be a promising marker for predicting the hematological adverse events of chemotherapy 
Table IV. Uni- and multivariate analysis for non-hematological toxicity.

\begin{tabular}{|c|c|c|c|c|}
\hline Variables & Unadjusted coef $(95 \% \mathrm{CI})$ & P-value & Adjusted coef $(95 \% \mathrm{CI})$ & P-value \\
\hline Male gender & $-0.326(-1.547$ to 0.895$)$ & 0.600 & $-0.745(-2.109$ to 0.619$)$ & 0.284 \\
\hline Age & $-0.007(-0.064$ to 0.049$)$ & 0.799 & $-0.044(-0.116$ to 0.027$)$ & 0.227 \\
\hline Rectum & $0.326(-0.895$ to 1.547$)$ & 0.600 & & \\
\hline Multiple metastasis & $0.481(-0.703$ to 1.666$)$ & 0.426 & 0.823 (-0.588 to 2.234$)$ & 0.253 \\
\hline Synchronous metastasis & $0.537(-0.648$ to 1.722$)$ & 0.374 & $1.146(-0.426$ to 2.719$)$ & 0.153 \\
\hline Number of cycles & $-0.094(-0.186$ to -0.003$)$ & 0.043 & $-0.127(-0.238$ to -0.017$)$ & 0.024 \\
\hline Use of bevacizumab & $-0.262(-1.444$ to 0.920$)$ & 0.664 & & \\
\hline Total serum bilirubin ${ }^{\mathrm{a}}$ & 0.508 (-1.538 to 2.554$)$ & 0.627 & $1.377(-1.298$ to 4.053$)$ & 0.313 \\
\hline $\mathrm{NFS}^{\mathrm{a}}$ & $0.053(-0.313$ to 0.419$)$ & 0.776 & $0.405(-0.101$ to 0.910$)$ & 0.117 \\
\hline
\end{tabular}

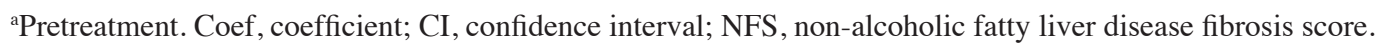

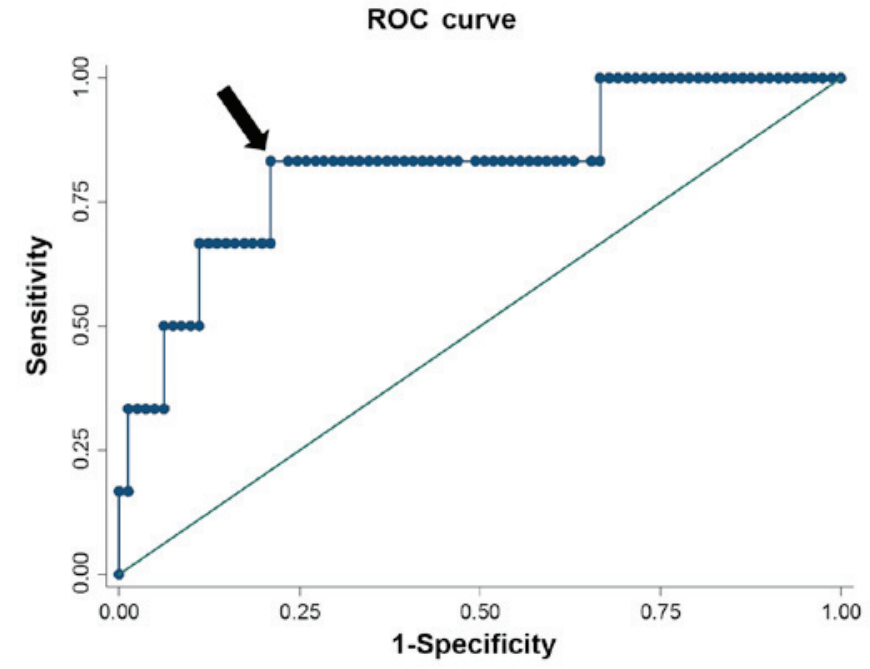

Figure 1. Receiver operating characteristic curve (ROC) analysis based on the association of pretreatment non-alcoholic fatty liver disease fibrosis score with hematological toxicity (Common Terminology Criteria for Adverse Effects grade $\geq 3$ ). Arrow, optimum cut-off point. In this model, the sensitivity was $83.3 \%$ and the specificity was $79.5 \%$.

including CPT-11 for colorectal cancer. Liver dysfunction, such as fibrosis, may cause severe CPT-11-related hematological adverse events by hampering its metabolism. The metabolism of CPT-11 occurs via a complex cascade, with hydrolysis of the parent prodrug by hepatic carboxylesterases into 7-ethyl-10-hydroxycamptothecin ( $\mathrm{SN}-38)$ as an active metabolite, detoxification of SN-38 into an inactive glucuronidated form (SN-38G) by UGT1A1, and excretion of SN-38G in the urine and bile (8). A fraction of SN-38 is also transported directly via ATP-binding cassette transporters into the bile and released in the intestinal lumen (9). In the intestine, SN-38 may undergo glucuronidation by intestinal UGT1A1 or be excreted unchanged in the feces (10). Thus, reduced SN-38G formation is closely associated with severe toxicities. As UGT1A1 is considered to be primarily responsible for the formation of SN-38G, genetic polymorphisms of UGT1A1, such as UGT $1 \mathrm{~A} 11^{*} 6$ and UGT $1 \mathrm{~A} 1^{*} 28$, increase CPT-11-related neutropenia $(6,11-13)$. UGT1A1 is also responsible for the detoxification of bilirubin, and a correlation between pretreatment bilirubin levels and UGT1A1 polymorphisms has been reported $(14,15)$. Thus, high levels of total bilirubin in the serum prior to treatment have been reported to be correlated with neutropenia $(12,13,16,17)$. However, this study did not determine the impact of pretreatment total bilirubin on the adverse effects of chemotherapy including CPT-11; instead, NFS was found to be a robust predictor of hematological adverse events. As the enzyme activity of UGT1A1 was reported to be largely unchanged by liver fibrosis in rats (18), other mechanisms through which liver dysfunction may cause CPT-11-related adverse effects have been suggested. However, CYP3A, one of the most important P450 enzymes, was dysregulated in those rats (19). Considering that bilirubin was not a significant predictor in our study, liver fibrosis may affect the metabolism of CPT-11, through mechanisms other than via UGT1A1, such as CYP3A4 or a bile duct transporter, and result in higher concentrations of SN-38.

Regarding non-hematological toxicity, the inactive metabolite $\mathrm{SN}-38 \mathrm{G}$ is secreted into the duodenum and deconjugated into $\mathrm{SN}-38$ by intestinal bacterial $\beta$-glucuronidase, which may cause diarrhea $(20,21)$. SN-38 is also converted into SN-38G, catalyzed by the UGT enzyme in the intestine (10). Thus, polymorphisms of UGT1A1 have been reported to increase not only neutropenia but also delayed diarrhea (22). NFS was not significantly associated with non-hematological toxicity in our findings, even when the investigation was limited to diarrhea, as liver fibrosis may not affect the activity of UGT or bacterial $\beta$-glucuronidase, at least in the intestine.

Our study revealed that NFS was a significant predictor of hematological toxicity in chemotherapy including CPT-11 for CRC. However, while some studies have pointed out the significance of chemotherapy-related adverse events, such as hematological toxicity, for the prognosis of colorectal cancer $(23,24)$, our study observed no correlation between NFS and prognosis (data not shown). This may explain why the impact of NFS was limited and why CPT-11 chemotherapy alone does not determine the prognosis of colorectal cancer patients, nor do pretreatment serum bilirubin levels or UGT1A1 polymorphisms $(16,25)$. Further investigation is required. 
This study has several limitations. Although the majority of studies on CPT-11 have included information on UGT1A1 polymorphisms, this was lacking in our medical records, as it is not routinely investigated at our institution. Another limitation was weakness of the statistical power of our study to a certain extent, as this was a retrospective study with a small sample size and data from a single institution. Thus, a prospective study with information on UGT1A1 polymorphisms should be conducted.

In conclusion, NFS is a significant predictor of hematological adverse events in chemotherapy including CPT-11 for colorectal cancer and its use may represent a non-invasive method useful for determining regimens or doses of chemotherapy including CPT-11.

\section{References}

1. Al Knawy B and Shiffman M: Percutaneous liver biopsy in clinical practice. Liver Int 27: 1166-1173, 2007.

2. Ratziu V, Charlotte F, Heurtier A, Gombert S, Giral P, Bruckert E, Grimaldi A, Capron F and Poynard T; LIDO Study Group: Sampling variability of liver biopsy in nonalcoholic fatty liver disease. Gastroenterology 128: 1898-1906, 2005.

3. McPherson S, Stewart SF, Henderson E, Burt AD and Day CP: Simple non-invasive fibrosis scoring systems can reliably exclude advanced fibrosis in patients with non-alcoholic fatty liver disease. Gut 59: 1265-1269, 2010.

4. Angulo P, Bugianesi E, Bjornsson ES, Charatcharoenwitthaya P, Mills PR, Barrera F, Haflidadottir S, Day CP and George J: Simple noninvasive systems predict long-term outcomes of patients with nonalcoholic fatty liver disease. Gastroenterology 145: 782-789. e4, 2013.

5. Yoneda M, Imajo K, Eguchi Y, Fujii H, Sumida Y, Hyogo H, Ono M, Suzuki Y, Kawaguchi T, Aoki N, et al: Noninvasive scoring systems in patients with nonalcoholic fatty liver disease with normal alanine aminotransferase levels. J Gastroenterol 48 : 1051-1060, 2013

6. Minami H, Sai K, Saeki M, Saito Y, Ozawa S, Suzuki K, Kaniwa N, Sawada J, Hamaguchi T, Yamamoto N, et al: Irinotecan pharmacokinetics/pharmacodynamics and UGT1A genetic polymorphisms in Japanese: Roles of UGT1A1" 6 and "28. Pharmacogenet Genomics 17: 497-504, 2007.

7. Cancer Therapy Evaluation Program (CTEP), N.C.I. Adverse Events (CTCAE) Version 4.0. 2009 June 14, 2010 [cited 2009 May 28]; Available from: http://ctep.cancer.gov/protocolDevelopment/electronic_applications/ctc.htm\#ctc_40.

8. Klein CE, Gupta E, Reid JM, Atherton PJ, Sloan JA, Pitot HC, Ratain MJ and Kastrissios H: Population pharmacokinetic model for irinotecan and two of its metabolites, $\mathrm{SN}-38$ and $\mathrm{SN}-38$ glucuronide. Clin Pharmacol Ther 72: 638-647, 2002.

9. Iyer L, King CD, Whitington PF, Green MD, Roy SK, Tephly TR, Coffman BL and Ratain MJ: Genetic predisposition to the metabolism of irinotecan (CPT-11). Role of uridine diphosphate glucuronosyltransferase isoform $1 \mathrm{~A} 1$ in the glucuronidation of its active metabolite (SN-38) in human liver microsomes. J Clin Invest 101: 847-854, 1998.

10. Hirose K, Kozu C, Yamashita K, Maruo E, Kitamura M, Hasegawa J, Omoda K, Murakami T and Maeda Y: Correlation between plasma concentration ratios of SN-38 glucuronide and SN-38 and neutropenia induction in patients with colorectal cancer and wild-type UGT1A1 gene. Oncol Lett 3: 694-698, 2012.

11. Innocenti F, Undevia SD, Iyer L, Chen PX, Das S, Kocherginsky M, Karrison T, Janisch L, Ramírez J, Rudin CM, et al: Genetic variants in the UDP-glucuronosyltransferase 1A1 gene predict the risk of severe neutropenia of irinotecan. J Clin Oncol 22: 1382-1388, 2004.
12. Onoue M, Terada T, Kobayashi M, Katsura T, Matsumoto S, Yanagihara K, Nishimura T, Kanai M, Teramukai S, Shimizu A, et al: UGT1A1*6 polymorphism is most predictive of severe neutropenia induced by irinotecan in Japanese cancer patients. Int J Clin Oncol 14: 136-142, 2009.

13. Ramchandani RP, Wang Y, Booth BP, Ibrahim A, Johnson JR, Rahman A, Mehta M, Innocenti F, Ratain MJ and Gobburu JV: The role of SN-38 exposure, UGT1A1*28 polymorphism, and baseline bilirubin level in predicting severe irinotecan toxicity. J Clin Pharmacol 47: 78-86, 2007.

14. Sai K, Saeki M, Saito Y, Ozawa S, Katori N, Jinno H, Hasegawa R, Kaniwa N, Sawada J, Komamura K, et al: UGT1A1 haplotypes associated with reduced glucuronidation and increased serum bilirubin in irinotecan-administered Japanese patients with cancer. Clin Pharmacol Ther 75: 501-515, 2004.

15. Bosma PJ, Saeki M, Saito Y, Ozawa S, Katori N, Jinno H, Hasegawa R, Kaniwa N, Sawada J and Komamura K: Bilirubin UDP-glucuronosyltransferase 1 is the only relevant bilirubin glucuronidating isoform in man. J Biol Chem 269: 17960-17964, 1994.

16. Meyerhardt JA, Kwok A, Ratain MJ, McGovren JP and Fuchs CS: Relationship of baseline serum bilirubin to efficacy and toxicity of single-agent irinotecan in patients with metastatic colorectal cancer. J Clin Oncol 22: 1439-1446, 2004.

17. Raymond E, Boige V, Faivre S, Sanderink GJ, Rixe O, Vernillet L, Jacques C, Gatineau M, Ducreux M and Armand JP: Dosage adjustment and pharmacokinetic profile of irinotecan in cancer patients with hepatic dysfunction. J Clin Oncol 20: 4303-4312, 2002.

18. Hao H, Zhang L, Jiang S, Sun S, Gong P, Xie Y, Zhou X and Wang G: Thioacetamide intoxication triggers transcriptional up-regulation but enzyme inactivation of UDP-glucuronosyltransferases.Drug MetabDispos 39: 1815-1822, 2011.

19. Xie Y, Wang G, Wang H, Yao X, Jiang S, Kang A, Zhou F, $\mathrm{Xie} \mathrm{T}$ and Hao H: Cytochrome P450 dysregulations in thioacetamide-induced liver cirrhosis in rats and the counteracting effects of hepatoprotective agents. Drug Metab Dispos 40: 796-802, 2012

20. Ikuno N, Soda H, Watanabe M and Oka M: Irinotecan (CPT-11) and characteristic mucosal changes in the mouse ileum and cecum. J Natl Cancer Inst 87: 1876-1883, 1995.

21. Takasuna K, Hagiwara T, Hirohashi M, Kato M, Nomura M, Nagai E, Yokoi T and Kamataki T: Involvement of beta-glucuronidase in intestinal microflora in the intestinal toxicity of the antitumor camptothecin derivative irinotecan hydrochloride (CPT-11) in rats. Cancer Res 56: 3752-3757, 1996.

22. Li M, Wang Z, Guo J, Liu J, Li C, Liu L, Shi H, Liu L, Li H, Xie C, et al: Clinical significance of UGT1A1 gene polymorphisms on irinotecan-based regimens as the treatment in metastatic colorectal cancer. Onco Targets Ther 7: 1653-1661, 2014.

23. Rambach L, Bertaut A, Vincent J, Lorgis V, Ladoire S and Ghiringhelli F: Prognostic value of chemotherapy-induced hematological toxicity in metastatic colorectal cancer patients. World J Gastroenterol 20: 1565-1573, 2014.

24. Shitara K, Matsuo K, Takahari D, Yokota T, Inaba Y, Yamaura H, Sato Y, Najima M, Ura T and Muro K: Neutropaenia as a prognostic factor in metastatic colorectal cancer patients undergoing chemotherapy with first-line FOLFOX. Eur J Cancer 45: 1757-1763, 2009.

25. Liu CY, Chen PM, Chiou TJ, Liu JH, Lin JK, Lin TC, Chen WS, Jiang JK, Wang HS and Wang WS: UGT1A1"28 polymorphism predicts irinotecan-induced severe toxicities without affecting treatment outcome and survival in patients with metastatic colorectal carcinoma. Cancer 112: 1932-1940, 2008. 